\title{
Existence of flows for linear Fokker-Planck-Kolmogorov equations and its connection to well-posedness
}

\section{MARCO REHMEIER(D}

Abstract. Let the coefficients $a_{i j}$ and $b_{i}, i, j \leq d$, of the linear Fokker-Planck-Kolmogorov equation (FPK-eq.)

$$
\partial_{t} \mu_{t}=\partial_{i} \partial_{j}\left(a_{i j} \mu_{t}\right)-\partial_{i}\left(b_{i} \mu_{t}\right)
$$

be Borel measurable, bounded and continuous in space. Assume that for every $s \in[0, T]$ and every Borel probability measure $v$ on $\mathbb{R}^{d}$ there is at least one solution $\mu=\left(\mu_{t}\right)_{t \in[s, T]}$ to the FPK-eq. such that $\mu_{s}=v$ and $t \mapsto \mu_{t}$ is continuous w.r.t. the topology of weak convergence of measures. We prove that in this situation, one can always select one solution $\mu^{s, v}$ for each pair $(s, v)$ such that this family of solutions fulfills

$$
\mu_{t}^{s, v}=\mu_{t}^{r, \mu_{r}^{s, v}} \text { for all } 0 \leq s \leq r \leq t \leq T,
$$

which one interprets as a flow property of this solution family. Moreover, we prove that such a flow of solutions is unique if and only if the FPK-eq. is well-posed.

\section{Introduction}

In this paper, we are concerned with linear Fokker-Planck-Kolmogorov equations of the form

$$
\partial_{t} \mu_{t}=\partial_{i} \partial_{j}\left(a_{i j} \mu_{t}\right)-\partial_{i}\left(b_{i} \mu_{t}\right)
$$

which are second-order parabolic equations for measures. Here, $a_{i j}, b_{i}:[0, T] \times$ $\mathbb{R}^{d} \rightarrow \mathbb{R}$ are given coefficients with suitable measurability and regularity assumptions, which are introduced below. For $s \in[0, T]$ and a Borel probability measure $v$ on $\mathbb{R}^{d}$, we consider the Cauchy problem of (1) with the initial condition $\mu_{s}=v$. Our notion of a solution to such a Cauchy problem is that of weakly continuous probability curves, i.e., a family of Borel probability measures $\left(\mu_{t}\right)_{t \in[s, T]}$ such that $[s, T] \ni t \mapsto \mu_{t}$ is weakly continuous and

$$
\int_{\mathbb{R}^{d}} f \mathrm{~d} \mu_{t}-\int_{\mathbb{R}^{d}} f \mathrm{~d} \nu=\int_{s}^{t} \int_{\mathbb{R}^{d}} L_{u} f(x) \mathrm{d} \mu_{u}(x) \mathrm{d} u
$$

Mathematics Subject Classification: 60J60, 35Q84

Keywords: Fokker-Planck-Kolmogorov equation, Flow property, Martingale problem, Superposition principle. 
holds for all smooth, compactly supported $f: \mathbb{R}^{d} \rightarrow \mathbb{R}$. A shorthand notation for the Cauchy problem of Eq. (1) is

$$
\left\{\begin{array}{l}
\partial_{t} \mu_{t}=L^{*} \mu_{t} \\
\mu_{s}=v
\end{array},\right.
$$

where $L^{*}$ denotes the formal dual of $L(t, x):=L_{A, b}(t, x):=\frac{1}{2} a_{i j}(t, x) \partial_{i} \partial_{j}+$ $b_{i}(t, x) \partial_{i}$, the second-order differential operator associated with $A:=\left(a_{i j}\right)_{i, j \leq d}$ and $b:=\left(b_{i}\right)_{i \leq d}$, also called Kolmogorov operator. Fokker-Planck-Kolmogorov equations have been an active research topic in the past decades. There is a vast literature on general results such as existence, uniqueness and regularity of solutions, also for a more general notion of equations than (1). A thorough analytical introduction to the field is provided by the work [1] of Röckner, Krylov, Bogachev and Shaposhnikov from 2015. Fokker-Planck-Kolmogorov equations also have strong and fruitful connections to probability theory, in particular to the theory of diffusion processes. For example, the transition probabilities of a typical diffusion process in $\mathbb{R}^{d}$ with drift $b=\left(b_{i}\right)_{i \leq d}$ and diffusion $\left(a_{i j}\right)_{i, j \leq d}$ solve the corresponding Fokker-Planck-Kolmogorov equation, i.e., Eq. (1). Above that, Eq. (1) is closely related to the martingale problem associated with the coefficients $a_{i j}$ and $b_{i}$. More precisely, every continuous solution to the martingale problem provides a weakly continuous probability solution to Eq. (1) via its one-dimensional marginals. Conversely, by a so-called superposition principle of Trevisan from 2016, which is an extension of an earlier work by Figalli from 2008, c.f. [2,5], for any weakly continuous probability solution $\left(\mu_{t}\right)_{t \in[s, T]}$ to Eq. (1), there exists a continuous solution to the corresponding martingale problem, for which the one-dimensional marginals are given by $\left(\mu_{t}\right)_{t \in[s, T]}$. A fundamental investigation of martingale problems and its connection to Fokker-Planck-Kolmogorov equations can be found in [4] by Stroock and Varadhan. In particular, in this work the authors prove the following: Given continuous and bounded coefficients $a_{i j}$ and $b_{i}$, assume there exists at least one continuous solution to the martingale problem with start in $x \in \mathbb{R}^{d}$ at time $s \geq 0$ for every pair $(s, x)$. Then, there exists a strong Markovian selection of such solutions. More precisely, one can select a solution $P^{s, x}$ to the martingale

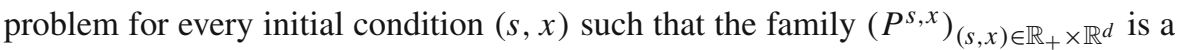
strong Markov process on the space of continuous functions $C\left(\mathbb{R}_{+}, \mathbb{R}^{d}\right)$. Such a consideration goes back to an earlier work of Krylov from 1973 (c.f. [3]). It is also proven that such a selection is unique if and only if the martingale problem is well-posed. The aim of this paper is to prove similar results for the Fokker-Planck-Kolmogorov equation (1). Our first main result (see Theorem 3.2) is the following: Assume that all coefficients $a_{i j}$ and $b_{i}$ are Borel measurable, bounded and continuous with respect to the spatial variables. Assuming that the Cauchy problem for Eq. (1) has at least one weakly continuous probability solution for every initial condition $(s, v)$, we prove the existence of a family of solutions $\left(\mu^{s, v}\right)_{s, v}$ such that 


$$
\mu_{t}^{s, v}=\mu_{t}^{r, \mu_{r}^{s, v}} \text { for all } 0 \leq s \leq r \leq t \leq T
$$

and each probability measure $v$ on $\mathbb{R}^{d}$. We interpret Eq. (2) as a flow property for solutions to (1). Moreover, in Theorem 3.16 we show: There exists exactly one such flow if and only if the Fokker-Planck-Kolmogorov equation is well-posed among weakly continuous probability solutions. The structure of this paper is as follows: In Sect. 2 we introduce notation, present the exact notion of a solution to the Cauchy problem of Eq. (1) and state the assumptions on the coefficients $a_{i j}$ and $b_{i}$. In the third section, we present our two main results, which are Theorems 3.2 and 3.16. We also set up all necessary notions and tools for its proofs. In particular, this includes the aforementioned superposition principle by Figalli and Trevisan. Afterward, we prove both main theorems. It would be interesting to generalize our results to FokkerPlanck-Kolmogorov equations on infinite-dimensional state spaces, e.g., replacing $\mathbb{R}^{d}$ by a separable Hilbert space $H$. The techniques we developed within the proof of Theorem 3.2 seem promising for such a generalization. In order to widen the spectrum of possible applications, it is also desirable to establish our main results under more general assumptions on the coefficients $a_{i j}$ and $b_{i}$. This could be a direction of further research on this topic.

\section{Preliminaries}

\subsection{Notation}

Let us introduce basic notation, which we will frequently use in the sequel. For a metric space $X, \mathcal{P}(X)$ denotes the set of all Borel probability measures on $X$. If $X=\mathbb{R}^{d}$, then we will simply write $\mathcal{P}:=\mathcal{P}\left(\mathbb{R}^{d}\right) . C_{b}\left(\mathbb{R}^{d}\right)$ is the set of all bounded and continuous functions $f: \mathbb{R}^{d} \rightarrow \mathbb{R}$ and $C_{c}^{\infty}\left(\mathbb{R}^{d}\right)$ the set of all such $f$, which are smooth and have compact support. For open sets $I \subseteq \mathbb{R}$ and $\Omega \subseteq \mathbb{R}^{d}, C_{b}^{1,2}(I \times \Omega)$ $\left[C_{c}^{1,2}(I \times \Omega)\right]$ denotes all bounded [compactly supported] functions $f: I \times \Omega \rightarrow \mathbb{R}$, which have at least one bounded continuous derivative w.r.t. $t \in I$ and at least two bounded continuous derivatives w.r.t. $x \in \Omega$. The spatial derivative in the $i$ th Euclidean direction for such a function is denoted by $\partial_{i} f, i \leq d$, and the derivative w.r.t. the timevariable by $\partial_{t} f$. As usual, for two topological spaces $X$ and $Y, C(X, Y)$ denotes the set of all continuous functions $f: X \rightarrow Y$. For a time interval $I \subseteq \mathbb{R}_{+}$, a Borel curve of Borel (probability) measures on $\mathbb{R}^{d}$ is a family $\left(\mu_{t}\right)_{t \in I}$ such that $t \mapsto \mu_{t}(A)$ is Borel measurable for every $A \in \mathcal{B}\left(\mathbb{R}^{d}\right)$. As usual, such a Borel curve is called weakly continuous, if $t \mapsto \int f \mathrm{~d} \mu_{t}$ is continuous for all $f \in C_{b}\left(\mathbb{R}^{d}\right)$, i.e., if the map $t \mapsto \mu_{t}$ is continuous w.r.t. the topology of weak convergence of measures on $\mathcal{P}$. The onedimensional Lebesgue measure on $\mathbb{R}$ or on an interval $I \subseteq \mathbb{R}$ is denoted by $\mathrm{d} t$. The set of all symmetric, nonnegative definite $d \times d$-matrices with real entries is denoted by $S_{+}\left(\mathbb{R}^{d}\right)$. 


\subsection{Basic setting}

Let $T>0$, which we fix throughout, and let

$$
a_{i j}, b_{i}:[0, T] \times \mathbb{R}^{d} \rightarrow \mathbb{R} \text { for } i, j \in\{1, \ldots, d\}
$$

fulfill the following assumptions: $a_{i j}$ and $b_{i}$ are Borel measurable, continuous in $x \in \mathbb{R}^{d}$ and globally bounded such that $A(t, x):=\left(a_{i j}(t, x)\right)_{i, j \leq d} \in S_{+}\left(\mathbb{R}^{d}\right)$ for every $(t, x) \in[0, T] \times \mathbb{R}^{d}$. These assumptions will be in force throughout the paper. Further, let $L:=L_{A, b}$ be the operator defined through $L f(t, x):=\frac{1}{2} a_{i j}(t, x) \partial_{i} \partial_{j} f(t, x)+$ $b_{i}(t, x) \partial_{i} f(t, x)$ for every $f: I \times \mathbb{R}^{d} \rightarrow \mathbb{R}$ with at least two spatial derivatives. If $f$ does not depend on $t \in I$, we write $L_{t} f(x)$ instead of $L f(t, x)$. We always assume summation over repeated indices.

Definition 2.1. A weakly continuous probability solution to the Cauchy problem for the Fokker-Planck-Kolmogorov equation (FPK-eq.) w.r.t. $a_{i j}, b_{i}$ on $[0, T] \times \mathbb{R}^{d}$ with the initial condition $(s, v) \in[0, T] \times \mathcal{P}$, i.e., to

$$
\left\{\begin{array}{l}
\partial_{t} \mu_{t}=\partial_{i} \partial_{j}\left(a_{i j} \mu_{t}\right)-\partial_{i}\left(b_{i} \mu_{t}\right) \\
\mu_{s}=v
\end{array}\right.
$$

sometimes shortly written as

$$
\left\{\begin{array}{l}
\partial_{t} \mu_{t}=L^{*} \mu_{t} \\
\mu_{s}=v
\end{array}\right.
$$

is a weakly continuous Borel curve of probability measures $\mu=\left(\mu_{t}\right)_{t \in[s, T]} \in$ $C([s, T], \mathcal{P})$ such that

$$
\int_{s}^{T} \int_{\mathbb{R}^{d}} \partial_{t} f(t, x)+L f(t, x) \mathrm{d} \mu_{t}(x) \mathrm{d} t=0
$$

holds for all $f \in C_{c}^{1,2}\left((s, T) \times \mathbb{R}^{d}\right)$ and $\mu_{s}=v$ is fulfilled. Equivalently, we may require

$$
\int_{\mathbb{R}^{d}} f \mathrm{~d} \mu_{t}-\int_{\mathbb{R}^{d}} f \mathrm{~d} \nu=\int_{s}^{t} \int_{\mathbb{R}^{d}} L_{u} f(x) \mathrm{d} \mu_{u}(x) \mathrm{d} u
$$

for all $f \in C_{c}^{\infty}\left(\mathbb{R}^{d}\right)$ and all $t \in[s, T]$.

Definition 2.2. The set of all weakly continuous probability solutions to the above FPK-eq. with the initial condition $(s, v)$ is denoted by $F P(L, s, v)$. Since we fix $L=L_{A, b}$ throughout, no confusion will occur when we write $F P(s, v)$ instead of $F P(L, s, v)$. 


\section{The main results}

Definition 3.1. Let $\gamma^{s, v}=\left(\gamma_{t}^{s, v}\right)_{t \in[s, T]} \in F P(s, v)$ for every $(s, v) \in[0, T] \times \mathcal{P}$. We say that the family $\left(\gamma^{s, v}\right)_{(s, v) \in[0, T] \times \mathcal{P}}$ has the flow property, if for every $0 \leq s \leq$ $r \leq t \leq T$ and $\nu \in \mathcal{P}$ we have

$$
\gamma_{t}^{s, v}=\gamma_{t}^{r, \gamma_{r}^{s, v}}
$$

Our first main result is the following statement.

Theorem 3.2. Assume $a_{i j}$ and $b_{i}$ fulfill the measurability and regularity conditions imposed in Definition 2.1. Further, assume that $F P(s, v)$ is non-empty for every $(s, v)$, i.e., for every initial condition $(s, v) \in[0, T] \times \mathcal{P}$ there exists a weakly continuous probability solution to the above FPK-eq. with start in $(s, v)$. Then, there exists a family of solutions $\left(\mu^{s, v}\right)_{(s, v) \in[0, T] \times \mathcal{P}}$ with the flow property.

For the proof, we need several preparations.

For $0 \leq s \leq T$, we set $\mathbb{Q}_{s}^{T}:=[s, T] \cap \mathbb{Q}$. Let $\mathcal{P}^{T}:=\left\{\left(\gamma_{q}\right)_{q \in \mathbb{Q}_{s}^{T}} \mid \gamma_{q} \in \mathcal{P}\right\}$ be endowed with the product topology of weak convergence of probability measures. This space is Polish, since the topology of weak convergence on $\mathcal{P}$ is metrizable by the Prohorov metric. Moreover, the space $C\left([s, T], \mathbb{R}^{d}\right)$ will always be endowed with the norm of uniform convergence.

Definition 3.3. Let $I$ be some index set. A family of measurable functions $\left\{f_{i}\right\}_{i \in I}$, $f_{i}: \mathbb{R}^{d} \rightarrow \mathbb{R}$, is called measure-determining (for finite Borel measures) on $\mathbb{R}^{d}$, if for any two finite Borel measures $\mu_{1}, \mu_{2}$ on $\mathbb{R}^{d}, \int f_{i} \mathrm{~d} \mu_{1}=\int f_{i} \mathrm{~d} \mu_{2}$ for all $i \in I$ implies $\mu_{1}=\mu_{2}$.

Remark 3.4. Clearly every dense, countable subset $\left\{f_{n}\right\}_{n \in \mathbb{N}}$ of $C_{c}^{\infty}\left(\mathbb{R}^{d}\right)$ is such a measure-determining family.

Definition 3.5. (i) We call a bijection $\eta: \mathbb{N} \times \mathbb{Q}_{0}^{T} \rightarrow \mathbb{N}_{0}$ an enumeration. Given an enumeration $\eta$, the $k$ th element in $\mathbb{N} \times \mathbb{Q}_{0}^{T}$ according to $\eta$ is denoted by $\left(n_{k}, q_{k}\right)$.

(ii) For $s \in[0, T]$, we denote by $\left(m_{k}^{s}\right)_{k \in \mathbb{N}_{0}}$ the enumerating sequence of $\mathbb{N} \times \mathbb{Q}_{s}^{T}$ according to $\eta$, i.e., $\eta^{-1}\left(m_{k}^{s}\right)$ is the $k$ th element in $\mathbb{N} \times \mathbb{Q}_{s}^{T}$ according to $\eta$.

Remark 3.6. Note that for $0 \leq s \leq r \leq T$, the sequence $\left(m_{l}^{r}\right)_{l \in \mathbb{N}_{0}}$ is a subsequence of $\left(m_{l}^{s}\right)_{l \in \mathbb{N}_{0}}$. This will be important when we verify the flow property in the proof of Theorem 3.2.

Definition 3.7. For $s \in[0, T]$ let $J_{s}: C([s, T], \mathcal{P}) \rightarrow \mathcal{P}^{\mathbb{Q}_{s}^{T}}$ be defined by

$$
J_{s}\left(\left(\gamma_{t}\right)_{t \in[s, T]}\right):=\left(\gamma_{q}\right)_{q \in \mathbb{Q}_{s}^{T}}
$$

i.e., it is the canonical projection on all coordinates $q \in \mathbb{Q}_{s}^{T}$. 
Remark 3.8. In the sequel we will always consider $C([s, T], \mathcal{P})$ with the product topology of weak convergence of probability measures and not, as common, with the topology of uniform convergence. Then, $J_{s}$ as in Definition 3.7 is clearly continuous for every $s \in[0, T]$. However, note that $C([s, T], \mathcal{P})$ endowed with the product topology of weak convergence of measures is not metrizable.

The next remark points out an important technique for the proof of Theorem 3.2.

Remark 3.9. By definition, we have $F P(s, v) \subseteq C([s, T], \mathcal{P})$ for all $s \in[0, T]$ and $v \in \mathcal{P}$. Since $\mathcal{P}$ is Polish and $[s, T]$ is compact, elements in $C([s, T], \mathcal{P})$ are even uniformly continuous and in particular every element in $F P(s, v)$ is uniquely determined by its values in a countable, dense subset of $[s, T]$. More precisely, for $\left(\gamma_{q}\right)_{q \in \mathbb{Q}_{s}^{T}} \in \mathcal{P}^{\mathbb{Q}_{s}^{T}}$, there is at most one $\left(\bar{\gamma}_{t}\right)_{t \in[s, T]} \in F P(s, v)$ such that $\bar{\gamma}_{q}=\gamma_{q}$ for all $q \in \mathbb{Q}_{S}^{T}$. The advantage of this consideration is that $\mathcal{P} \mathbb{Q}_{s}^{T}$ is, in contrast to $C([s, T], \mathcal{P})$, metrizable and hence continuity of a map $f: \mathcal{P}^{\mathbb{Q}_{s}^{T}} \rightarrow \mathbb{R}$ is equivalent to its sequential continuity.

Before we turn to the proof of Theorem 3.2, we need to introduce further additional important results about Fokker-Planck-Kolmogorov equations and their connection to the corresponding martingale problem. We start by recalling the definition of a solution to the martingale problem associated with given coefficients $a_{i j}$ and $b_{i}$.

Definition 3.10. Let $L=L_{A, b}$ denote the differential operator associated with the given coefficients $a_{i j}$ and $b_{i}$ from above as introduced in the previous section. A (continuous) solution to the martingale problem associated with $L$ with the initial condition $(s, v) \in[0, T] \times \mathcal{P}$ is a measure $P \in \mathcal{P}\left(C\left([s, T], \mathbb{R}^{d}\right)\right)$ such that

(i) $P \circ \pi_{s}^{-1}=v$

(ii) For every $\phi \in C_{b}^{1,2}\left((s, T) \times \mathbb{R}^{d}\right)$, the process $[s, T] \ni t \mapsto \phi\left(t, \pi_{t}\right)-$ $\int_{s}^{t} \partial_{u} \phi(u, \cdot) \circ \pi_{u}+L \phi\left(u, \pi_{u}\right) \mathrm{d} u$ is a real-valued $P$-martingale on $C\left([s, T], \mathbb{R}^{d}\right)$ w.r.t. the natural filtration $\left(\mathcal{F}_{t}\right)_{t \in[s, T]}$ on $C\left([s, T], \mathbb{R}^{d}\right)$, i.e., $\mathcal{F}_{t}:=\sigma\left(\pi_{u} \mid u \in\right.$ $[s, t])$ for all $t \in[s, T]$.

Here and below, $\pi_{t}: C\left([s, T], \mathbb{R}^{d}\right) \rightarrow \mathbb{R}^{d}$ denotes the canonical projection at time $t \in[s, T]$.

Proposition 3.11. Let $P \in \mathcal{P}\left(C\left([s, T], \mathbb{R}^{d}\right)\right)$ be a solution to the martingale problem associated with $L$ with the initial condition $(s, v)$. Then, $\left(P \circ \pi_{t}^{-1}\right)_{t \in[s, T]} \in F P(s, v)$. Proof. Obviously, $\left(P \circ \pi_{t}^{-1}\right)_{t \in[s, T]}$ is a Borel curve of probability measures. Due to the continuity of the canonical projections $\pi_{t}$ for $t \in[s, T],\left(P \circ \pi_{t}^{-1}\right)_{t \in[s, T]}$ is clearly weakly continuous. Using the martingale property of Definition 3.10 (ii), we obtain by integration with respect to $P$, Fubini's theorem, a change of variables for image measures and (i) of the previous definition:

$$
\int_{\mathbb{R}^{d}} \phi \mathrm{d} P_{t}-\int_{s}^{t} \int_{\mathbb{R}^{d}} L_{u} \phi \mathrm{d} P_{u} \mathrm{~d} u=\int_{\mathbb{R}^{d}} \phi \mathrm{d} P_{s}=\int_{\mathbb{R}^{d}} \phi \mathrm{d} v
$$

for every $\phi \in C_{c}^{\infty}\left(\mathbb{R}^{d}\right)$, where we abbreviated $P \circ \pi_{t}^{-1}$ by $P_{t}$. 
The following theorem by Trevisan gives sort of an inverse to the above proposition. We point out that Trevisan's result (c.f. Theorem 2.5 in [5]), which is an extension of an earlier work by Figalli (c.f. [2]), does not require any continuity or boundedness of the coefficients (instead of the latter, in [5] global integrability of $a_{i j}$ and $b_{i}$ w.r.t. $\mathrm{d} \gamma_{t}(x) \mathrm{d} t$ over $[s, T] \times \mathbb{R}^{d}$ is required for any solution $\left.\gamma\right)$.

Theorem 3.12. (Superposition principle by Trevisan/Figalli) Let $\left(\gamma_{t}\right)_{t \in[s, T]} \in F P$ $(s, v)$. Then, there exists a solution $P \in \mathcal{P}\left(C\left([s, T], \mathbb{R}^{d}\right)\right)$ to the martingale problem associated with $L$ with start in $(s, v)$ such that $P \circ \pi_{t}^{-1}=\gamma_{t}$ holds for all $t \in[s, T]$.

The following proposition is a minor extension of Theorem 1.4.6 in [4] and provides a convenient tool to check whether a given family $\mathcal{M} \subseteq \mathcal{P}\left(C\left([s, T], \mathbb{R}^{d}\right)\right)$ is precompact w.r.t. the topology of weak convergence of probability measures. Below, $B_{l}(x) \subseteq \mathbb{R}^{d}$ denotes the Euclidean ball with radius $l \geq 0$ centered around $x \in \mathbb{R}^{d}$. For $f: \mathbb{R}^{d} \rightarrow \mathbb{R}$ and $a \in \mathbb{R}^{d}, f_{a}(x):=f(x-a)$ is the translate of $f$ by $a \in \mathbb{R}^{d}$.

Proposition 3.13. A family $\mathcal{M} \subseteq \mathcal{P}\left(C\left([s, T], \mathbb{R}^{d}\right)\right)$ is precompact if and only if both of the following conditions hold:

(i) $\lim _{l \rightarrow+\infty} \sup _{P \in \mathcal{M}} P \circ \pi_{s}^{-1}\left(B_{l}(0)^{c}\right)=0$

(ii) For every nonnegative $f \in C_{c}^{\infty}\left(\mathbb{R}^{d}\right)$, there exists a constant $c_{f} \geq 0$, which does not depend on $P \in \mathcal{M}$, such that $f_{a}\left(\pi_{t}\right)+c_{f} t$ is a nonnegative submartingale w.r.t. the natural filtration on $C\left([s, T], \mathbb{R}^{d}\right)$ for every $P \in \mathcal{M}$ and every $a \in \mathbb{R}^{d}$.

Proof. It suffices to note that the proof of Theorem 1.4.6. in [4] still holds when one replaces $C\left(\mathbb{R}_{+}, \mathbb{R}^{d}\right)$ by $C\left([s, T], \mathbb{R}^{d}\right)$ for arbitrary $0 \leq s \leq T<+\infty$.

We now state a crucial compactness result for the set of solutions to the martingale problem associated with $L$ with the initial condition $(s, v)$. Essentially, this result is formulated as part of Lemma 12.2.1 in [4]. However, as this lemma only covers the compactness for deterministic initial conditions-i.e., $v=\delta_{x}$ for $x \in \mathbb{R}^{d}$-in the case of time-independent coefficients, for the convenience of the reader we decided to give a proof for the more general version, which we shall need below.

Proposition 3.14. Let $M P(s, v) \subseteq \mathcal{P}\left(C\left([s, T], \mathbb{R}^{d}\right)\right)$ be the set of all continuous solutions to the martingale problem associated with $L$ with the initial condition $(s, v)$. Then, $M P(s, v)$ is a compact subset of $\mathcal{P}\left(C\left([s, T], \mathbb{R}^{d}\right)\right)$ (which is endowed with the topology of weak convergence of measures).

Proof. Using Proposition 3.13, we first show that $M P(s, v)$ is precompact. Indeed, since $P \circ \pi_{s}^{-1}=v$ holds for all $P \in M P(s, v)$ and since every Borel probability measure on $\mathbb{R}^{d}$ is tight, we obtain $(i)$ of Proposition 3.13. Concerning ( $\left.i i\right)$, note that for nonnegative $f \in C_{c}^{\infty}\left(\mathbb{R}^{d}\right)$, due to the boundedness of $a_{i j}$ and $b_{i}$ and since $f$ is compactly supported, there is a constant $c_{f} \geq 0$ such that

$$
L_{u} f\left(\pi_{u}\right) \geq-c_{f}
$$


for all $u \in[s, T]$. Hence, $[s, T] \ni t \mapsto \int_{s}^{t} L_{u} f\left(\pi_{u}\right) \mathrm{d} u+c_{f} t$ is nonnegative and increasing and thus for any $P \in M P(s, v)$ the process

$$
f\left(\pi_{t}\right)+c_{f} t=f\left(\pi_{t}\right)-\int_{s}^{t} L_{u} f\left(\pi_{u}\right) \mathrm{d} u+\int_{s}^{t} L_{u} f\left(\pi_{u}\right) \mathrm{d} u+c_{f} t
$$

is the sum of a martingale and a nonnegative, increasing adapted process and is therefore a nonnegative submartingale on $[s, T]$ w.r.t. the natural filtration on $C\left([s, T], \mathbb{R}^{d}\right)$ under $P$. It is clear that the same constant $c_{f}$ works for all translates $f_{a}$ as well, since the boundedness of all coefficients and $f \in C_{c}^{\infty}\left(\mathbb{R}^{d}\right)$ yields that (5) holds for every $f_{a}$. Hence, Proposition 3.13 applies and $M P(s, v) \subseteq \mathcal{P}\left(C\left([s, T], \mathbb{R}^{d}\right)\right)$ is precompact.

It remains to show closedness of $M P(s, v)$. Therefore, let $\left\{P_{n}\right\}_{n \in \mathbb{N}} \subseteq M P(s, v)$ such that $P_{n} \underset{n \rightarrow+\infty}{\rightarrow} P$ weakly in $\mathcal{P}\left(C\left([s, T], \mathbb{R}^{d}\right)\right)$. First of all, it is obvious that we have $P \circ \pi_{s}^{-1}=v$, since the canonical projection at time s, $\pi_{s}: C\left([s, T], \mathbb{R}^{d}\right) \rightarrow \mathbb{R}^{d}$, is continuous and $P_{n} \circ \pi_{s}^{-1}=v$ holds for all $n \in \mathbb{N}$. In order to prove $P \in M P(s, v)$, we show

$$
\begin{aligned}
& \int\left[\phi\left(t, \pi_{t}\right)-\int_{s}^{t} \partial_{u} \phi(u, \cdot) \circ \pi_{u}+L \phi\left(u, \pi_{u}\right) \mathrm{d} u\right] G_{r} \mathrm{~d} P \\
& =\int\left[\phi\left(r, \pi_{r}\right)-\int_{s}^{r} \partial_{u} \phi(u, \cdot) \circ \pi_{u}+L \phi\left(u, \pi_{u}\right) \mathrm{d} u\right] G_{r} \mathrm{~d} P
\end{aligned}
$$

for all $0 \leq s \leq r \leq t \leq T, \phi \in C_{b}^{1,2}\left((s, T) \times \mathbb{R}^{d}\right)$ and every continuous, bounded $\mathcal{F}_{r}$-measurable $G_{r}: C\left([s, T], \mathbb{R}^{d}\right) \rightarrow \mathbb{R}$. But due to $P_{n} \in M P(s, v)$, the above equality holds for $P$ replaced by any $P_{n}$. Further,

$$
\left(\phi\left(z, \pi_{z}\right)-\int_{s}^{z} \partial_{u} \phi(u, \cdot) \circ \pi_{u}+L \phi\left(u, \pi_{u}\right) \mathrm{d} u\right) G_{r}: C\left([s, T], \mathbb{R}^{d}\right) \rightarrow \mathbb{R}
$$

is bounded and continuous for every $z \in[s, T], G_{r}$ and $\phi$ : Indeed, boundedness is immediate due to the boundedness of $G_{r}, \phi$ and the coefficients $a_{i j}, b_{i}$ and continuity follows, because the integrand of the parameter-dependent integral

$$
C\left([s, T], \mathbb{R}^{d}\right) \ni \zeta \mapsto \int_{s}^{z} \partial_{u} \phi(u, \cdot) \circ \pi_{u}(\zeta)+L \phi\left(u, \pi_{u}(\zeta)\right) \mathrm{d} u
$$

is integrable for fixed $\zeta$, continuous in $\zeta$ for fixed $u \in[s, z]$ and bounded in $\zeta$ and $u$ by a constant (the latter due to the boundedness of $a_{i j}, b_{i}$ and $\phi$ ). Therefore, the parameter-dependent integral is continuous and the weak convergence $P_{n} \underset{n \rightarrow+\infty}{\rightarrow} P$ implies the desired equality. Thus, $P \in M P(s, v)$ follows, which completes the proof.

We now turn to the proof of Theorem 3.2. We point out that a similar method of proof can be traced back at least to the work of Krylov ([3]), who considered a similar, yet not identical, selection method to construct a Markovian selection from what he calls a Markovian system of processes. Later, Stroock and Varadhan used 
a reminiscent approach to prove the existence of a strong Markovian selection to the martingale problem associated with continuous and bounded coefficients in $\mathbb{R}^{d}$ (c.f. Chapter 12 in [4]). However, since neither of these works considers a selection method on the level of the Fokker-Planck-Kolmogorov equation, our presentation below differs from these seminal results. Naturally, our selection method is adapted to select a unique curve of probability measures rather than selecting a measure on path space. To the best of our knowledge, such a selection method has not been carried out for Fokker-Planck-Kolmogorov equations before. Nevertheless, a careful comparison of our technique below and the works cited above will reveal a principal reminiscence.

Proof of Theorem 3.2. Let $\left\{f_{n}\right\}_{n \in \mathbb{N}} \subseteq C_{b}\left(\mathbb{R}^{d}\right)$ be a measure-determining family and $\eta$ a fixed enumeration, as presented in Definition 3.5. Below we adopt the notation of Definition 3.5. Further, fix $(s, v) \in[0, T] \times \mathcal{P}$. We define the following values, maps and sets, where for abbreviation we occasionally write $\mu$ instead of $\left(\mu_{q}\right)_{q \in \mathbb{Q}_{s}^{T}}$. The map $J_{S}$ is as introduced in Definition 3.7.

$$
\begin{aligned}
u_{m_{0}^{s}}(s, v) & :=\sup _{\mu \in J_{s}(F P(s, v))} \int f_{n_{m_{0}^{s}}} \mathrm{~d} \mu_{q_{m_{0}^{s}}}, \\
G_{m_{0}^{s}}^{s, v}: J_{s}(F P(s, v)) & \rightarrow \mathbb{R},\left(\mu_{q}\right)_{q \in \mathbb{Q}_{s}^{T}} \mapsto \int f_{n_{m_{0}^{s}}} \mathrm{~d} \mu_{q_{m_{0}^{s}}}, \\
M_{m_{0}^{s}}(s, v) & :=G_{m_{0}^{s}}^{s, v-1}\left(\left\{u_{m_{0}^{s}}\right\}\right)
\end{aligned}
$$

and iteratively for $k \in \mathbb{N}_{0}$

$$
\begin{aligned}
u_{m_{k+1}^{s}}(s, v) & :=\sup _{\mu \in M_{m_{k}^{s}}(s, v)} \int f_{n_{m_{k+1}^{s}}} \mathrm{~d} \mu_{q_{m_{k+1}^{s}}}, \\
G_{m_{k+1}^{s}}^{s, v}: M_{m_{k}^{s}}(s, v) & \rightarrow \mathbb{R},\left(\mu_{q}\right)_{q \in \mathbb{Q}_{s}^{T}} \mapsto \int f_{n_{m_{k+1}^{s}}} \mathrm{~d} \mu_{q_{m_{k+1}^{s}}}, \\
M_{m_{k+1}^{s}}(s, v) & :=G_{m_{k+1}^{s}, v}-1\left(\left\{u_{m_{k+1}^{s}}\right\}\right) .
\end{aligned}
$$

We make the following observations: Since we assume $F P(s, v) \neq \varnothing$ for all $(s, v) \in$ $[0, T] \times \mathcal{P}$, we have $J_{s}(F P(s, v)) \neq \emptyset$. Therefore, and because each $f_{n}$ is bounded and for $\mu \in J_{s}(F P(s, v))$ every marginal $\mu_{q}$ is a probability measure, we have $u_{m_{0}^{s}}(s, v) \in$ $\mathbb{R}$. By the same argument, we have $u_{m_{k+1}^{s}}(s, v) \in \mathbb{R}$ for $k \in \mathbb{N}_{0}$, provided $M_{m_{k}^{s}} \neq \emptyset$. Moreover, $G_{m_{0}^{s}}^{s, v}$ is continuous, because $\left(\mu_{q}\right)_{q \in \mathbb{Q}_{s}^{T}} \mapsto \mu_{q_{m_{0}^{s}}}$ is clearly continuous from $J_{S}(F P(s, v))$ with the induced product topology of weak convergence to $\mathcal{P}$ with the topology of weak convergence and $\mu_{q_{m_{0}^{s}}} \mapsto \int f_{n_{m_{0}^{s}}} \mathrm{~d} \mu_{q_{m_{0}^{s}}}$ is continuous by definition of the topology of weak convergence of measures and since $f_{n} \in C_{b}\left(\mathbb{R}^{d}\right)$ for every $n \in \mathbb{N}$. If $M_{m_{k}^{s}} \neq \varnothing$, then the same holds true for $G_{m_{k+1}^{s}}^{s, v}$. 
Our aim is to prove $\left|\bigcap_{k \in \mathbb{N}_{0}} M_{m_{k}^{s}}(s, v)\right|=1$. Naturally we prove this in two steps. We start by proving $\left|\bigcap_{k \in \mathbb{N}_{0}} M_{m_{k}^{s}}(s, v)\right| \leq 1$ : If

$$
\left(\mu_{q}^{1}\right)_{q \in \mathbb{Q}_{s}^{T}},\left(\mu_{q}^{2}\right)_{q \in \mathbb{Q}_{s}^{T}} \in \bigcap_{k \in \mathbb{N}_{0}} M_{m_{k}^{s}}(s, v),
$$

then, since $\left\{m_{k}^{s} \mid k \in \mathbb{N}_{0}\right\}$ is an enumerating sequence of $\mathbb{N} \times \mathbb{Q}_{s}^{T}$, we have $\int f_{n} \mathrm{~d} \mu_{q}^{1}=$ $\int f_{n} \mathrm{~d} \mu_{q}^{2}$ for all $(n, q) \in \mathbb{N} \times \mathbb{Q}_{s}^{T}$. Since $\left\{f_{n}\right\}_{n \in \mathbb{N}}$ is measure-determining, we obtain $\mu_{q}^{1}=\mu_{q}^{2}$ for all $q \in \mathbb{Q}_{s}^{T}$, which implies $\left|\bigcap_{k \in \mathbb{N}_{0}} M_{m_{k}^{s}}(s, v)\right| \leq 1$.

By definition of $M_{m_{0}^{s}}(s, v)$, both $\left(\mu_{q}^{1}\right)_{q \in \mathbb{Q}_{s}^{T}}$ and $\left(\mu_{q}^{2}\right)_{q \in \mathbb{Q}_{s}^{T}}$ are elements of $J_{s}(F P$ $(s, v))$, and thus, there is a unique element $\left(\bar{\mu}_{t}\right)_{t \in[s, T]} \in F P(s, v)$ such that $\mu_{q}^{1}=$ $\bar{\mu}_{q}=\mu_{q}^{2}$ for all $q \in \mathbb{Q}_{s}^{T}$. We conclude: There exists at most one element $\mu=$ $\left(\mu_{t}\right)_{t \in[s, T]} \in F P(s, v)$ such that $\left(\mu_{q}\right)_{q \in \mathbb{Q}_{s}^{T}} \in J_{s}(F P(s, v))$ (see also Remark 3.9).

We now show $\left|\bigcap_{k \in \mathbb{N}_{0}} M_{m_{k}^{s}}(s, v)\right| \geq 1$ : We start by proving compactness of $J_{s}(F P$ $(s, v)) \subseteq \mathcal{P}^{\mathbb{Q}_{s}^{T}}$

By Proposition 3.14, $M P(s, v)$ is a compact subset of $\mathcal{P}\left(C\left([s, T], \mathbb{R}^{d}\right)\right)$ with the topology of weak convergence. Now define the following map (we abbreviate $Q_{t}:=$ $\left.Q \circ \pi_{t}^{-1}\right)$ :

$$
\Lambda: \mathcal{P}\left(C\left([s, T], \mathbb{R}^{d}\right)\right) \rightarrow C([s, T], \mathcal{P}), \quad Q \mapsto\left(Q \circ \pi_{t}^{-1}\right)_{t \in[s, T]} .
$$

To prove the continuity of $\Lambda$, suppose that $Q^{n} \underset{n \rightarrow+\infty}{\rightarrow} Q$ in $\mathcal{P}\left(C\left([s, T], \mathbb{R}^{d}\right)\right)$, i.e., $\int G d Q^{n} \underset{n \rightarrow+\infty}{\rightarrow} \int G d Q$ for all $G \in C_{b}\left(C\left([s, T], \mathbb{R}^{d}\right)\right)$. Clearly, for every $g \in$ $C_{b}\left(\mathbb{R}^{d}\right)$ and $t \in[s, T]$, we have $g \circ \pi_{t} \in C_{b}\left(C\left([s, T], \mathbb{R}^{d}\right)\right)$ and hence in particular $\int g d Q_{t}^{n} \underset{n \rightarrow+\infty}{\rightarrow} \int g d Q_{t}$ holds for all such $g$ and $t$, which implies $\left(Q_{t}^{n}\right)_{t \in[s, T]} \underset{n \rightarrow+\infty}{\rightarrow}$ $\left(Q_{t}\right)_{t \in[s, T]}$ in $C([s, T], \mathcal{P})$ (endowed with the product topology of weak convergence of measures) and thus the continuity of $\Lambda$. Hence, $\Lambda(M P(s, v)) \subseteq C([s, T], \mathcal{P})$ is compact. By Proposition 3.11 (giving “ $\subseteq$ ”) and Theorem 3.12 (giving “ $\supseteq$ "), we deduce

$$
\Lambda(M P(s, v))=F P(s, v)
$$

and therefore compactness of $F P(s, v) \subseteq C([s, T], \mathcal{P})$.

By Remark 3.8 and the above considerations, the map

$$
J_{s} \circ \Lambda: \mathcal{P}\left(C\left([s, T], \mathbb{R}^{d}\right)\right) \rightarrow \mathcal{P}^{\mathbb{Q}_{s}^{T}}, \quad Q \mapsto\left(Q \circ \pi_{q}^{-1}\right)_{q \in \mathbb{Q}_{s}^{T}}
$$

is continuous as well. Therefore and by (6), $J_{s}(F P(s, v)) \subseteq \mathcal{P} \mathbb{Q}_{s}^{T}$ is compact. Thus, by the continuity of $G_{m_{0}^{s}}^{s, v}, M_{m_{0}^{s}}(s, v) \subseteq \mathcal{P}^{\mathbb{Q}_{s}^{T}}$ is non-empty and compact. Repeating the same arguments with $J_{s}(F P(s, v))$ replaced by $M_{m_{0}^{s}}(s, v), G_{m_{0}^{s}}^{s, v}$ by $G_{m_{1}^{s}}^{s, v}$ and 
$u_{m_{0}^{s}}(s, v)$ by $u_{m_{1}^{s}}(s, v)$, we obtain that $M_{m_{1}^{s}}(s, v) \subseteq M_{m_{0}^{s}}(s, v)$ is non-empty and compact as well. Iterating this procedure, we obtain a sequence of non-empty, compact sets $\left(M_{m_{k}^{s}}(s, v)\right)_{k \in \mathbb{N}_{0}}$ with $M_{m_{k+1}^{s}}(s, v) \subseteq M_{m_{k}^{s}}(s, v) \subseteq \mathcal{P}^{\mathbb{Q}_{s}^{T}}$ for all $k \in \mathbb{N}_{0}$. Finally, this implies $\left|\bigcap_{k \in \mathbb{N}_{0}} M_{m_{k}^{s}}(s, v)\right| \geq 1$. Let us recapitulate what we have achieved so far: For each initial condition $(s, v) \in[0, T] \times \mathcal{P}$, we have characterized a unique element $\mu^{s, v}=\left(\mu_{t}^{s, v}\right)_{t \in[s, T]} \in F P(s, v)$ in the sense that it is the unique element in $F P(s, v)$ such that $\left(\mu_{q}^{s, \nu}\right)_{q \in \mathbb{Q}_{s}^{T}} \in \bigcap_{k \in \mathbb{N}_{0}} M_{m_{k}^{s}}(s, v)$. We consider $\mu^{s, v}$ as an extremal element in $F P(s, v)$, because by construction it is "iteratively maximal" in the sense that $\left(\mu_{q}^{s, v}\right)_{q \in \mathbb{Q}_{s}^{T}} \in M_{m_{k}^{s}}$ for every $k \in \mathbb{N}_{0}$.

To conclude the proof, it remains to show that the family $\left(\mu^{s, v}\right)_{(s, v) \in[0, T] \times \mathcal{P}}$ has the desired flow property, i.e., that it fulfills (4) of Definition 3.1. In order to prove this, let us fix $0 \leq s \leq r \leq T$ and $v \in \mathcal{P}$ and let $\mu^{s, v}=\left(\mu_{t}^{s, v}\right)_{t \in[s, T]} \in F P(s, v)$ be the unique iteratively maximal solution for the initial condition $(s, v)$ as selected in the previous passage. Consider the initial condition $\left(r, \mu_{r}^{s, \nu}\right)$ and let $\gamma=\left(\gamma_{t}\right)_{t \in[r, T]} \in F P\left(r, \mu_{r}^{s, v}\right)$ be the unique iteratively maximal solution for the initial condition $\left(r, \mu_{r}^{s, v}\right)$, i.e., in our notation $\gamma_{t}=\mu_{t}^{r, \mu_{r}^{s, v}}$. We need to show

$$
\gamma_{t}=\mu_{t}^{s, v} \text { for all } t \in[r, T] .
$$

We proceed as follows: Define $\zeta=\left(\zeta_{t}\right)_{t \in[s, T]}$ by

$$
\zeta_{t}:= \begin{cases}\mu_{t}^{s, \nu}, & \text { if } t \in[s, r] \\ \gamma_{t}, & \text { if } t \in[r, T]\end{cases}
$$

which is a well-defined Borel curve of probability measures, since $\gamma_{r}=\mu_{r}^{s, \nu}$ and since both $\mu^{s, v}$ and $\gamma$ are Borel curves. Clearly $\zeta \in F P(s, v)$ : Indeed, it is obvious that $[s, T] \ni t \mapsto \zeta_{t}$ is weakly continuous, that $\zeta_{s}=v$ holds and for $f \in C_{c}^{\infty}\left(\mathbb{R}^{d}\right)$ and $t \in[s, T]$ we have

$$
\begin{aligned}
\int f \mathrm{~d} \zeta_{t}-\int f \mathrm{~d} v & =\int f \mathrm{~d} \zeta_{t}-\int f \mathrm{~d} \mu_{r}^{s, v}+\int f \mathrm{~d} \mu_{r}^{s, v}-\int f \mathrm{~d} v \\
& =\int_{r}^{t} \int_{\mathbb{R}^{d}} L_{u} f(x) \mathrm{d} \gamma_{u}(x) \mathrm{d} u+\int_{s}^{r} \int_{\mathbb{R}^{d}} L_{u} f(x) \mathrm{d} \mu_{u}^{s, v}(x) \mathrm{d} u \\
& =\int_{s}^{t} \int_{\mathbb{R}^{d}} L_{u} f(x) \mathrm{d} \zeta_{u} \mathrm{~d} u, \text { if } t \in[r, T]
\end{aligned}
$$

and

$$
\int f \mathrm{~d} \zeta_{t}-\int f \mathrm{~d} \nu=\int_{s}^{t} \int_{\mathbb{R}^{d}} L_{u} f(x) \mathrm{d} \mu_{u}^{s, v} \mathrm{~d} u=\int_{s}^{t} \int_{\mathbb{R}^{d}} L_{u} f(x) \mathrm{d} \zeta_{u} \mathrm{~d} u, \text { if } t \in[s, r],
$$

which implies $\zeta \in F P(s, v)$ by Definition 2.1. Therefore, by virtue of the characterizing property of $\mu^{s, v}$ among all elements of $F P(s, v)$, we have

$$
\int f_{n_{m_{0}}} \mathrm{~d} \mu_{q_{m_{0}^{s}}}^{s, v} \geq \int f_{n_{m_{0}^{s}}} \mathrm{~d} \zeta_{q_{m_{0}}}
$$


If $q_{m_{0}^{s}} \in[s, r]$, then $\zeta_{q_{0}^{s}}=\mu_{q_{m_{0}^{s}}}^{s, v}$ and we have equality in (8). If $\left.\left.q_{m_{0}^{s}} \in\right] r, T\right]$, then $q_{m_{0}^{s}}=q_{m_{0}^{r}}$ and by the characterizing property of $\gamma$ among all elements of $F P\left(r, \mu_{r}^{s, \nu}\right)$ and since $\left(\mu_{t}^{s, v}\right)_{t \in[r, T]} \in F P\left(r, \mu_{r}^{s, v}\right)$, we have

$$
\int f_{n_{m_{0}}} \mathrm{~d} \mu_{q_{m_{0}}^{s}}^{s, \nu} \leq \int f_{n_{m_{0}^{s}}} \mathrm{~d} \gamma_{q_{m_{0}}}=\int f_{n_{m_{0}^{s}}} \mathrm{~d} \zeta_{q_{m_{0}}}
$$

and hence we have equality in (8) in any case. Now consider $m_{1}^{s}$ : Since we have equality in (8), both $\left(\mu_{q}^{s, v}\right)_{q \in \mathbb{Q}_{s}^{T}}$ and $\left(\zeta_{q}\right)_{q \in \mathbb{Q}_{s}^{T}}$ belong to $M_{m_{0}^{s}}(s, v)$. Hence, using the characterization of $\mu^{s, v}$ again, we obtain

$$
\int f_{n_{m_{1}^{s}}} \mathrm{~d} \mu_{q_{m_{1}^{s}}}^{s, v} \geq \int f_{n_{m_{1}^{s}}} \mathrm{~d} \zeta_{q_{m_{1}^{s}}}
$$

clearly with equality if $q_{m_{1}^{s}} \in[s, r]$. If $\left.\left.q_{m_{1}^{s}} \in\right] r, T\right]$ and $q_{m_{0}^{s}} \in[s, r]$, then $m_{1}^{s}=m_{0}^{r}$ and we must have

$$
\int f_{n_{m_{1}^{s}}} \mathrm{~d} \mu_{q_{m_{1}^{s}}^{s}}^{s, v} \leq \int f_{n_{m_{1}^{s}}} \mathrm{~d} \gamma_{q_{m_{1}^{s}}}=\int f_{n_{m_{1}^{s}}} \mathrm{~d} \zeta_{q_{m_{1}^{s}}}
$$

by the characterizing property of $\gamma$ and hence equality in (9). If $\left.\left.q_{m_{0}^{s}}, q_{m_{1}^{s}} \in\right] r, T\right]$, then $m_{0}^{s}=m_{0}^{r}, m_{1}^{s}=m_{1}^{r}$ and both $\left(\mu_{q}^{s, \nu}\right)_{q \in \mathbb{Q}_{r}^{T}}$ and $\left(\gamma_{q}\right)_{q \in \mathbb{Q}_{r}^{T}}$ are in $M_{m_{0}^{r}}\left(r, \mu_{r}^{s, v}\right)$ and we also obtain (10). Hence, equality in (9) holds in any case. Iterating this procedure yields

$$
\int f_{n_{m_{k}}} \mathrm{~d} \mu_{q_{m_{k}^{s}}^{s}}^{s, v}=\int f_{n_{m_{k}}} \mathrm{~d} \zeta_{q_{m_{k}}}
$$

for all $k \in \mathbb{N}_{0}$. As $\left(m_{k}^{s}\right)_{k \in \mathbb{N}_{0}}$ is the enumerating sequence of $\mathbb{N} \times \mathbb{Q}_{s}^{T}$ and $\left\{f_{n}\right\}_{n \in \mathbb{N}}$ is measure-determining on $\mathbb{R}^{d}$, this yields

$$
\mu_{q}^{s, v}=\zeta_{q} \text { for all } q \in \mathbb{Q}_{s}^{T}
$$

so in particular $\mu_{q}^{s, v}=\gamma_{q}$ for all $q \in \mathbb{Q}_{r}^{T}$. Since both $\left(\gamma_{q}\right)_{q \in \mathbb{Q}_{r}^{T}}$ and $\left(\mu_{q}^{s, v}\right)_{q \in \mathbb{Q}_{r}^{T}}$ belong to $J_{r}\left(F P\left(r, \mu_{r}^{s, v}\right)\right)$, we obtain (7).

Remark 3.15. We point out that if we perform the selection method of the above proof for a different measure-determining family $\left\{g_{n}\right\}_{n \in \mathbb{N}} \subseteq C_{b}\left(\mathbb{R}^{d}\right)$ or with a different enumeration $\delta$ instead of $\eta$, we may obtain a different family of solutions fulfilling the flow property. This also becomes apparent in the next theorem and its proof. Above that, in principle, one could also consider a different dense, countable subset of $[0, T]$ instead of $\mathbb{Q}_{0}^{T}$. This will, in principle, also lead to a different selected solution family.

The following theorem is an interesting consequence of the method we used to construct a flow of solutions within the proof of Theorem 3.2.

Theorem 3.16. Let all assumptions of Theorem 3.2 be in force. Then, the following assertions are equivalent: 
(i) The FPK-eq. is well-posed among weakly continuous probability solutions, i.e., $|F P(s, v)|=1$ for all $(s, v) \in[0, T] \times \mathcal{P}$.

(ii) There exists a unique family of solutions $\left(\mu^{s, v}\right)_{(s, v) \in[0, T] \times \mathcal{P}}$ with the flow property.

Proof. The implication $(i) \Longrightarrow$ (ii) follows immediately, because the existence of a flow follows by Theorem 3.2 and due to the assumption on well-posedness, there can obviously not be two differing flows.

Concerning $(i i) \Longrightarrow(i)$ assume, under the general assumption $|F P(s, v)| \geq 1$ for all $(s, v) \in[0, T] \times \mathcal{P}$, that the FPK-eq. is not well-posed, i.e., there exists an initial condition $(\bar{s}, \bar{v})$ such that $|F P(\bar{s}, \bar{v})| \geq 2$. Let $\left\{f_{n}\right\}_{n \in \mathbb{N}} \subseteq C_{b}\left(\mathbb{R}^{d}\right)$ be a measuredetermining family to which with each $f_{n}$ also $-f_{n}$ belongs, $\eta: \mathbb{N} \times \mathbb{Q}_{0}^{T} \rightarrow \mathbb{N}_{0}$ an enumeration and let $u_{m_{k}^{s}}(s, v)$ and $M_{m_{k}^{s}}(s, v)$ be defined as in the proof of Theorem 3.2 for all $k \in \mathbb{N}_{0}, s \in[0, T]$ and $v \in \mathcal{P}$. Let $\left(\mu^{s, v}\right)_{(s, v) \in[0, T] \times \mathcal{P}}$ be the selected flow subject to this data as constructed in the proof of Theorem 3.2.

By assumption, for $(\bar{s}, \bar{v})$ there is $\gamma \in F P(\bar{s}, \bar{v})$ such that $\gamma_{\bar{t}} \neq \mu_{\bar{t}}^{\bar{s}, \bar{v}}$ for some $\bar{t} \in$ $[s, T]$. By weak continuity of elements in $F P(s, v)$, we may without loss of generality assume $\bar{t} \in \mathbb{Q} \frac{T}{s}$. Hence, there must be a member of the measure-determining family, say $f_{\bar{n}}$, such that

$$
\int f_{\bar{n}} \mathrm{~d} \mu_{\bar{t}}^{\bar{s}, \bar{v}} \neq \int f_{\bar{n}} \mathrm{~d} \gamma_{\bar{t}} .
$$

Let us without loss of generality assume

$$
\int f_{\bar{n}} \mathrm{~d} \gamma_{\bar{t}}>\int f_{\bar{n}} \mathrm{~d} \mu_{\bar{t}}^{\bar{s}, \bar{v}},
$$

else we consider $-f_{\bar{n}}$ instead, which by assumption also belongs to the family $\left\{f_{n}\right\}_{n \in \mathbb{N}}$.

Now consider the same measure-determining sequence $\left\{f_{n}\right\}_{n \in \mathbb{N}}$, but a different enumeration $\delta$ such that according to this enumeration $\left(f_{n_{0}}, q_{n_{0}}\right)=\left(f_{\bar{n}}, \bar{t}\right)$ and denote the corresponding flow of solutions constructed as in the proof of Theorem 3.2 by

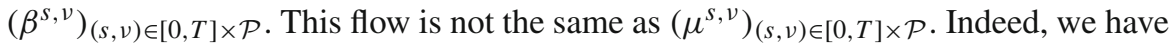

$$
\int f_{\bar{n}} \mathrm{~d} \beta_{\bar{t}}^{\bar{s}, \bar{v}} \geq \int f_{\bar{n}} \mathrm{~d} \gamma_{\bar{t}}>\int f_{\bar{n}} \mathrm{~d} \mu_{\bar{t}}^{\bar{s}, \bar{v}} .
$$

Here, the first inequality holds by the characterizing property of $\beta^{\bar{s}, \bar{v}}$ and the choice of the enumeration $\delta$ and the second one is due to assumption (11). Hence, we cannot have

$$
\int f_{\bar{n}} \mathrm{~d} \mu_{\bar{t}}^{\bar{s}, \bar{v}}=\int f_{\bar{n}} \mathrm{~d} \beta_{\bar{t}}^{\bar{s}, \bar{v}}
$$

and thereby $\mu_{\bar{t}}^{\bar{s}, \bar{v}} \neq \beta_{\bar{t}}^{\bar{s}, \bar{v}}$ follows, which shows that the two flows $\left(\mu^{s, v}\right)_{(s, v) \in[0, T] \times \mathcal{P}}$ and $\left(\beta^{S, \nu}\right)_{(s, \nu) \in[0, T] \times \mathcal{P}}$ are not identical. This finishes the proof. 
Remark 3.17. We would like to point out the following observation: From the proof of Theorem 3.2, it is clear that instead of $F P(s, v)$, we can also consider arbitrary closed, non-empty subsets $C(s, v) \subseteq F P(s, v)$ with the following property: Whenever $\left(\gamma_{t}\right)_{t \in[s, T]} \in C(s, v)$, then $\left(\gamma_{t}\right)_{t \in[r, T]} \in C\left(r, \gamma_{r}\right)$ and for $\gamma^{1} \in F P(s, v)$ and $\gamma^{2} \in$ $F P\left(r, \gamma_{r}^{1}\right)$, the curve

$$
\rho_{t}:= \begin{cases}\gamma_{t}^{1}, & \text { if } t \in[s, r[ \\ \gamma_{t}^{2}, & \text { if } t \in[r, T]\end{cases}
$$

belongs to $F P(s, v)$ for all $0 \leq s \leq r \leq T$ and $v \in \mathcal{P}$. Performing the same method as in the proof of Theorem 3.2, we then construct a family of solutions $\left(\mu^{s, v}\right)_{(s, v) \in[0, T] \times \mathcal{P}}$ with the flow property and $\mu^{s, v} \in C(s, v)$ for every $(s, v)$. This could provide a useful tool to a priori restrict the set of solutions within which we select the members of our flow. It is then obvious that also Theorem 3.16 holds when each $F P(s, v)$ is replaced by such $C(s, v)$.

\section{Acknowledgements}

Open Access funding provided by Projekt DEAL. I am indebted to my supervisor Prof. Dr. Michael Röckner, who pointed out this interesting topic to me and with whom I shared various fruitful discussions concerning the proof of the first main theorem. Furthermore, financial support by the German Science Foundation DFG (IRTG 2235) is gratefully acknowledged.

Open Access. This article is licensed under a Creative Commons Attribution 4.0 International License, which permits use, sharing, adaptation, distribution and reproduction in any medium or format, as long as you give appropriate credit to the original author(s) and the source, provide a link to the Creative Commons licence, and indicate if changes were made. The images or other third party material in this article are included in the article's Creative Commons licence, unless indicated otherwise in a credit line to the material. If material is not included in the article's Creative Commons licence and your intended use is not permitted by statutory regulation or exceeds the permitted use, you will need to obtain permission directly from the copyright holder. To view a copy of this licence, visit http://creativecommons.org/licenses/ by $/ 4.0 /$.

Publisher's Note Springer Nature remains neutral with regard to jurisdictional claims in published maps and institutional affiliations.

\section{REFERENCES}

[1] V.I. Bogachev, N.V. Krylov, M. Röckner, and S.V. Shaposhnikov. Fokker-Planck-Kolmogorov Equations. Mathematical Surveys and Monographs. American Mathematical Society, 2015.

[2] A. Figalli. Existence and uniqueness of martingale solutions for sdes with rough or degenerate coefficients. Journal of Functional Analysis, 254(1):109 - 153, 2008.

[3] N. V. Krylov. On the selection of a markov process from a system of processes and the construction of quasi-diffusion processes. 7, 7(3):691-709, jun 1973.

[4] D.W. Stroock and S.R.S. Varadhan. Multidimensional Diffusion Processes. Classics in Mathematics. Springer Berlin Heidelberg, 2007. 
[5] D. Trevisan. Well-posedness of multidimensional diffusion processes with weakly differentiable coefficients. Electron. J. Probab., 21:41 pp., 2016.

Marco Rehmeier
Faculty of Mathematics
Bielefeld University
33615 Bielefeld
Germany

E-mail:mrehmeier@math.uni-bielefeld.de 